\title{
Preoperative High-Resolution Ultrasound for the Assessment of Macro-Metastatic Central Lymph Nodes Sublevel-By-Sublevel in Unilateral Papillary Thyroid Microcarcinoma
}

\section{Wen Tan}

Sun Yat-sen University Cancer Center

\section{Lingli Xiao}

Sun Yat-sen University Cancer Center

\section{Shiwen Zhang}

Sun Yat-sen University Cancer Center Haoming Zheng

Sun Yat-sen University Cancer Center

Sheng Li

Sun Yat-sen University Cancer Center

Yu Zhang

Sun Yat-sen University Cancer Center

Ankui Yang

Sun Yat-sen University Cancer Center

Jianhua Zhou

Sun Yat-sen University Cancer Center

Longzhong Liu ( $\sim$ liulzh@sysucc.org.cn )

Sun Yat-sen University Cancer Center https://orcid.org/0000-0003-4639-1767

Research article

Keywords: Papillary Thyroid Microcarcinoma, Central Lymph Node, Micrometastasis, Ultrasonic Diagnosis

Posted Date: February 18th, 2022

DOl: https://doi.org/10.21203/rs.3.rs-746447/v1

License: (c) (i) This work is licensed under a Creative Commons Attribution 4.0 International License. Read Full License 


\section{Abstract}

Background To understand the diagnostic efficacy of preoperative high-resolution ultrasound (HRUS) in the diagnosis of macro-metastatic central lymph node (macro-CLNM), It will help surgeons decide whether to perform prophylactic lymph nodes (LNs) dissection in patients with papillary thyroid microcarcinoma (PTMC).

Methods This study analyzed the preoperative ultrasound and postoperative pathological results of central lymph nodes sublevel-by-sublevel of 220 patients with unilateral PTMC. These patients all underwent surgery at the Sun Yat-sen University Cancer Center from January 2015 to November 2019.

Results In the right paratracheal, the left paratracheal, the pretracheal, and the prelaryngeal level, the mean number of lymph nodes are $0.9,1.9,3.3$, and 4.6 , respectively, and the macro-metastasis rate are $0.91 \%, 6.36 \%, 6.73 \%$, and $11.21 \%$, respectively. For metastasis central lymph nodes (CLNM), the HRUS diagnostic efficacy in the four sublevels was low, with the AUC of 0.50-0.54. When the diagnosis object was changed from CLNM to macro-CLNM, the AUC of the ipsilateral paratracheal sublevel has been significantly improved, with $0.80(95 \% \mathrm{Cl} 0.71-0.87)$ on the left and $0.75(95 \% \mathrm{Cl} 0.67-0.83)$ on the right. Meanwhile, the negative predictive value (NPV) of ultrasound diagnosis of macrometastasis in ipsilateral paratracheal sublevel reached $99 \%$ and $95 \%$.

Conclusions The number and macro-metastasis rate of lymph nodes in PTMC patients are, from high to low, the right paratracheal, the left paratracheal, the pretracheal, and the prelaryngeal level. Preoperative HRUS has a high diagnostic efficiency for macro-CLNM in the ipsilateral paratracheal area; when the ultrasound diagnosis of the ipsilateral paratracheal area is negative, the probability of macrometastasis is extremely low.

\section{Introduction}

The global incidence rate of thyroid cancer has increased rapidly by 3-15 times since the 1980 s. Among them, the incidence rate of large papillary thyroid cancer (PTC) and other pathological types of thyroid cancer has remained the same compared with that before 1980 . The vast majority of rapidly increasing cases are small papillary thyroid carcinoma (less than $15 \mathrm{~mm}$ ), half of which are papillary thyroid microcarcinoma (PTMC) ${ }^{1,2}$, defined as the longest diameter less than $10 \mathrm{~mm}$, regardless of whether there is lymph node metastasis ${ }^{3}$.

Generally, PTMC progresses slowly and has a good prognosis, but it is more often accompanied by central lymph node metastasis (CLNM) ${ }^{4-7}$, which is also proved to be an independent risk factor for recurrence in patients with thyroid cancer ${ }^{8-11}$. The 2015 ATA guideline suggested ${ }^{12}$ that thyroidectomy without prophylactic central neck dissection may be is appropriate for small (T1 or T2), noninvasive, clinically node-negative PTC (cNO) (Strong Recommendation). However, according to the guideline, the patients with thyroid cancer accompanied by macro-metastatic cervical lymph nodes (the cancer foci in 
the lymph node longer than $2 \mathrm{~mm}^{13}$ ) have an intermediate risk of recurrence. It means that, it is necessary to perform central neck dissection even for PTMC patients if preoperative high-resolution ultrasound (HRUS) detects macro-metastatic central lymph nodes (macro-CLNM). Otherwise, there will be an increasing rate for the second surgery. Therefore, understanding the diagnostic efficacy of preoperative HRUS in the diagnosis of macro-CLNM will help surgeons decide whether to perform prophylactic lymph nodes (LNs) dissection in patients with PTMC. Although several studies indicate that preoperative ultrasound has low diagnostic efficiency for CLNM of PTMC ${ }^{5,14}$, whether the diagnostic efficacy of HRUS for macro-CLNM is also very low, there has not been any literature report as far as we know.

American Joint Committee on Cancer staging system ${ }^{15}$ (AJCC, 8th edition) divides central lymph nodes (LNs) into four sublevels, including prelaryngeal LNs, pretracheal LNs, the left and the right paratracheal LNs. The dissection scope of central LNs for PTMC consisted of the LNs in prelaryngeal, pretracheal, and at least ipsilateral paratracheal sublevel ${ }^{16}$. Although it is difficult to correspond the cervical LNs detected by HRUS with that in pathology one by one, if the central compartment can be subdivided, and the LNs can be corresponded in ultrasonic examinations and pathology according to the four sublevels, the evaluation of HRUS diagnostic efficacy will be more accuracy.

To sum up, this study covered 220 consecutive PTMC patients, comparing their preoperative ultrasound and postoperative pathology of the central LNs in each sublevel to figure out the macro-CLNM rates and the diagnostic efficacy of preoperative ultrasound in each sublevel. It will be a reference for developing individualized surgical plans for PTMC patients.

\section{Methods}

This study was approved by our Institutional Review Board, and the requirement for informed consent was waived because of its retrospective nature. This article's authenticity was validated by uploading the key raw data onto the Research Data Deposit public platform (www.researchdata.org.cn), with the Research Data Deposit number RDDA2020001813.

\section{Patients}

In order to reduce the interference of the contralateral central lymph node and lateral cervical lymph node metastasis on the analysis of the ultrasound diagnosis performance in each sublevel, this study analyzed the preoperative ultrasound and postoperative pathological results of 220 patients with unilateral PTMC. These patients all underwent surgery at the Sun Yat-sen University Cancer Center from January 2015 to November 2019. The inclusion criteria are as follows: 1) PTMC Patients whose lymph nodes were nopalpable by physical examination; 2)Unilateral PTMC was confirmed by postoperative pathology; the surgery method was thyroidectomy total or lobectomy and ipsilateral central lymph node dissection; 3 ) preoperative HRUS examination and postoperative pathological results were reported sublevel-bysublevel. Patients were excluded if they met the following criteria: have received treatment before, with 
contralateral central or lateral cervical lymph node dissection, with distant metastases, were followed-up for less than one year, recurrence was found during the follow-up, had incomplete pathological data.

\section{Preoperative ultrasound examination and diagnosis}

Patients included in this study underwent ultrasound examinations one week before the surgery, using a high-resolution sonography system (GE, Mindray, SIEMENS, Toshiba, Super Sonic, Hitachi, PHILIPS, etc.) equipped with an at least $10 \mathrm{MHz}$ linear array transducer. Central compartment (level VI) lymph nodes were divided into prelaryngeal, pretracheal, and ipsilateral paratracheal sublevel by AJCC (8th edition). During the preoperative ultrasound examination, the largest and/or the most suspicious LNs detected in each sublevel should be marked down, and the following sonographic features of lymph nodes should be described: the length of the long and short axis, the shape (oval, round, or irregular), the margin (regular or irregular), corticomedullary demarcations (clear or unclear), hilar structure (detected or not detected) and blood flow signals (hilar, peripheral, or mixed type). Besides, for suspicious LNs, the features supporting the diagnosis of metastasis should be recorded. The 2015 ATA guidelines ${ }^{12}$ pointed out that the cervical LNs should be considered metastatic if the following sonographic features occurred: round shape, microcalcifications, cystic aspect, hyperechogenicity, and peripheral vascularization.

\section{Surgery and pathological data}

All patients included in this study underwent unilateral central compartment lymph nodes dissection, and the surgical area covered from prelaryngeal level, pretracheal level to ipsilateral paratracheal level, and the excised lymph nodes were marked by sublevels and sent for pathological examination. By reviewing the pathological slices, two experienced pathologists divided the central LNs in each sublevel into no, micro (the cancer foci was shorter than $2 \mathrm{~mm}$ ), and macro-CLNM (the cancer foci was $2 \mathrm{~mm}$ or longer than $2 \mathrm{~mm}$ ) according to the classification of metastatic LNs in the 8 th $\mathrm{JJCC}^{13}$. In this study: $\otimes$ a sublevel was

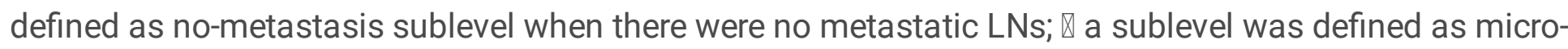
metastasis sublevel when there was one or more micro-metastatic LNs without any macro-metastatic

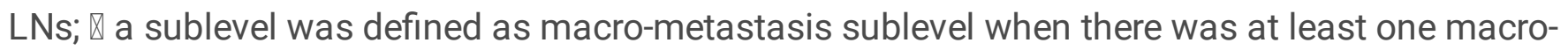
metastatic LN. If the two pathologists had different opinions on the diagnosis of the LNs, the two will give a consensus diagnosis after consultation.

\section{Statistical analysis}

We used MedCalc ${ }^{\circledR}$ statistical software to analyze the statistics. The independent-samples t-test was used to compare continuous variables. Categorical variables were compared using the Chi-squared $\left(\chi^{2}\right)$ test. We calculated the ultrasound diagnostic accuracy (ACC), sensitivity (SENS), specificity (SPEC), positive predictive value (PPV), negative predictive value (NPV), and the area under the ROC curve (AUC) to find out the ultrasound diagnostic efficacy for macro-CLNM or CLNM in each sublevel. P-values $<0.05$ were considered indicative of a statistically significant difference.

\section{Results}


Among the 220 patients with PTMC included in this study, 217 cases were pathologically confirmed as classical variants, while 3 cases were follicular variants. There were 95 patients with metastatic central compartment LNs, which accounted for about $43 \%$. The included cases were divided into 220 sublevels of prelaryngeal LNs, 220 sublevels of pretracheal LNs, 104 sublevels of left paratracheal LNs, and 116 sublevels of right paratracheal LNs (Table 1).

Table 1

Patient demographics and clinicopathological variables $(\mathrm{n}=$ 220)

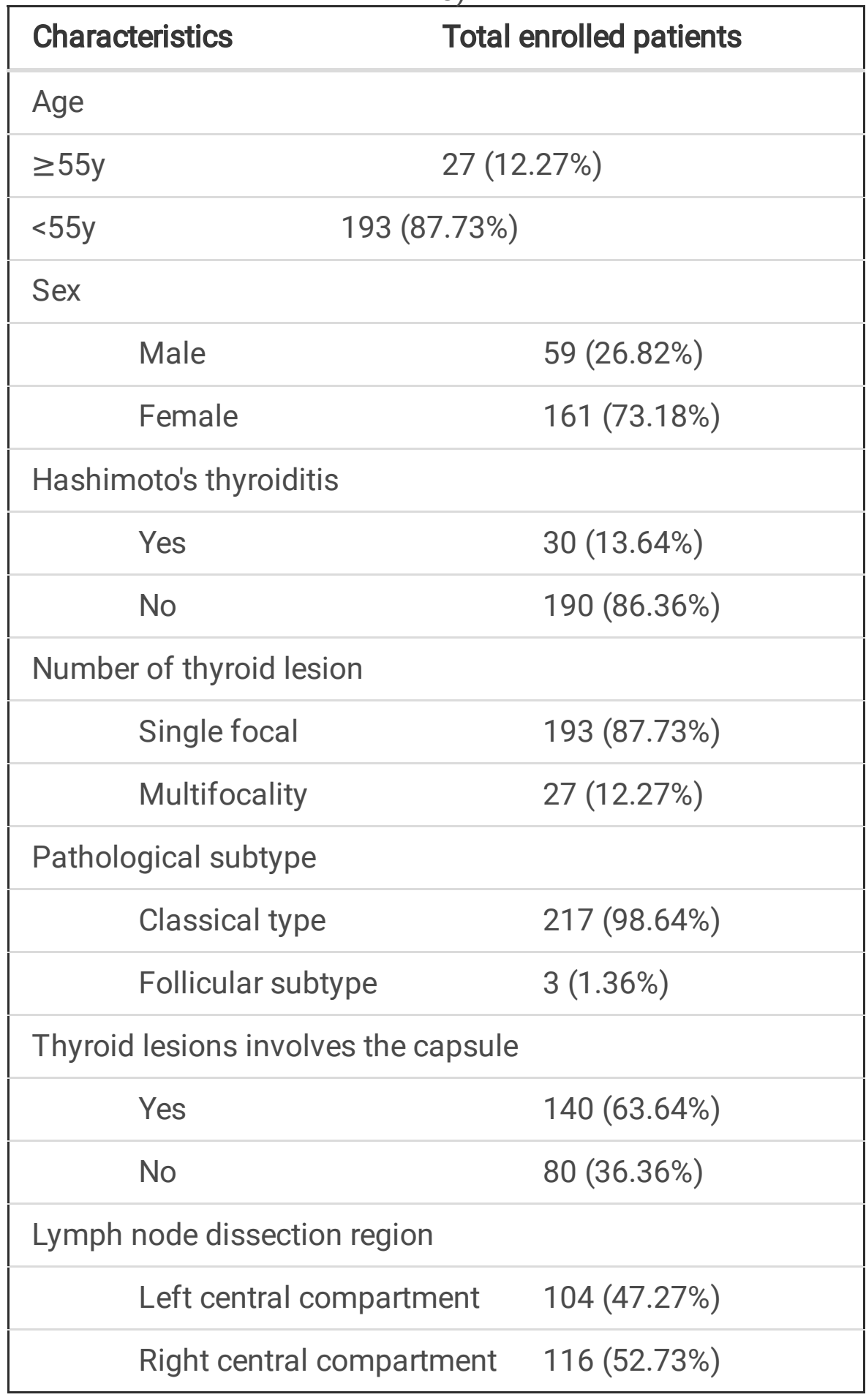




\section{The number of LNs and macro-metastasis rate in each sublevel according to pathological results}

The postoperative pathological results (Table 2 and Table 3 ) showed a tiny number of prelaryngeal LNs, with only $188 \mathrm{LNs}$ removed in 220 patients. Meanwhile, the LN macro-metastasis rate in prelaryngeal sublevel was also the lowest among all sublevels, only about $0.91 \%$. Next was the pretracheal LNs, with an average number of about $1.9 \pm 0.1 \mathrm{LNs}$ per case; the macro-metastasis rate in the pretracheal sublevel was higher than that of the prelaryngeal LNs, reaching $6.36 \%$. It was worth mentioning that the number of LNs in the right paratracheal was significantly beyond that in the left $(P<0.0001)$, whose average values were $4.6 \pm 0.3$ and $3.3 \pm 0.2$, respectively. The macro-metastasis rate of the right paratracheal LNs was also higher than that of the left side, accounting for approximately $11.21 \%$ and $6.73 \%$, respectively.

Table 2

The average number of central lymph nodes sublevel-by-sublevel on pathology in PTMC

\begin{tabular}{|lll|}
\hline & Total number of lymph nodes & Average number of lymph nodes \\
\hline Prelaryngeal LNs $(\mathrm{n}=220)$ & 188 & $0.9 \pm 0.1$ \\
\hline Pretracheal LNs $(\mathrm{n}=220)$ & 417 & $1.9 \pm 0.1$ \\
\hline Left paratracheal LNs $(\mathrm{n}=104)$ & 339 & $3.3 \pm 0.2$ \\
\hline Right paratracheal LNs $(\mathrm{n}=116)$ & 533 & $4.6 \pm 0.3$ \\
\hline PTMC, Papillary thyroid microcarcinoma; LNs, Lymph nodes.
\end{tabular}

Table 3

The rate of no-CLNM, micro-CLNM, and macro-CLNM in each sublevel.

\begin{tabular}{|c|c|c|c|}
\hline & \multicolumn{2}{|c|}{$\begin{array}{l}\text { The sublevel of central compartment } \\
\text { without macro-metastasis }\end{array}$} & \multirow{2}{*}{$\begin{array}{l}\text { The sublevel of centra } \\
\text { compartment with } \\
\text { macro-metastasis }\end{array}$} \\
\hline & no-metastasis & micro-metastasis & \\
\hline $\begin{array}{l}\text { Prelaryngeal LNs }(n= \\
220)\end{array}$ & $93.64 \%(206)$ & $5.45 \%(12)$ & $0.91 \%(2)$ \\
\hline $\begin{array}{l}\text { Pretracheal LNs }(n= \\
220)\end{array}$ & $82.27 \%(181)$ & $11.36 \%(25)$ & $6.36 \%(14)$ \\
\hline $\begin{array}{l}\text { Left paratracheal LNs } \\
(n=104)\end{array}$ & $69.23 \%(72)$ & $24.04 \%(25)$ & $6.73 \%(7)$ \\
\hline $\begin{array}{l}\text { Right paratracheal } \\
\text { LNs }(n=116)\end{array}$ & $63.79 \%(74)$ & $25.00 \%(29)$ & $11.21 \%(13)$ \\
\hline
\end{tabular}

The size and the HRUS detective rate of macro-metastatic LNs in each sublevel. 
As shown in the Table 4, among the 660 sublevels, the detective rate was extremely low $(0-21 \%)$ in the prelaryngeal and pretracheal sublevels no matter there was metastatic LNs or not. However, as for ipsilateral paratracheal sublevels, the detective rate of macro-CLNM was higher than non-macro-CLNM, with the macro-CLNM detective rate in left and right paratracheal of $85.71 \%$ and $76.92 \%$, respectively, the non-macro-CLNM detective rate of $38.14 \%$ and $32.04 \%$, respectively. However, there was no statistical significance in the HRUS detective rate of no- and micro-CLNM in the left and right paratracheal sublevels. In Table 5, the mean long-axis diameter of the macro-CLNM in the prelaryngeal, pretracheal, left paratracheal, and right paratracheal was $4 \mathrm{~mm}, 5.4 \mathrm{~mm}, 4.3 \mathrm{~mm}$, and $4.9 \mathrm{~mm}$, respectively; the mean longaxis diameter of the non-macro-CLNM was $3 \mathrm{~mm}, 3.5 \mathrm{~mm}, 3.6 \mathrm{~mm}$, and $3.1 \mathrm{~mm}$, respectively. the long and short-axis diameters of the macro-CLNM were longer than that of the non-macro-CLNM in each sublevel, and there was statistical significance in the pretracheal and left paratracheal sublevels $(P<0.05)$.

Table 4

The ultrasonic detecting rate of lymph nodes in each sublevel.

\begin{tabular}{|c|c|c|c|c|}
\hline & \multicolumn{2}{|c|}{$\begin{array}{l}\text { The sublevel of central compartment } \\
\text { without macro-metastasis }\end{array}$} & \multirow{2}{*}{$\begin{array}{l}\text { The sublevel of central } \\
\text { compartment with } \\
\text { macro-metastasis }\end{array}$} & \multirow[t]{2}{*}{$\begin{array}{l}P \text { - } \\
\text { value }\end{array}$} \\
\hline & no-metastasis & micro-metastasis & & \\
\hline $\begin{array}{l}\text { Prelaryngeal LNs } \\
(n=220)\end{array}$ & $1.46 \%(3 / 206)$ & $0 \%(0 / 12)$ & $0 \%(0 / 2)$ & - \\
\hline $\begin{array}{l}\text { Pretracheal LNs ( } \mathrm{n} \\
=220 \text { ) }\end{array}$ & $7.18 \%(13 / 181)$ & $8 \%(2 / 25)$ & $21.43 \%(3 / 14)$ & 0.003 \\
\hline $\begin{array}{l}\text { Left paratracheal } \\
\text { LNs }(n=104)\end{array}$ & $40.28 \%(29 / 72)$ & $32 \%(8 / 25)$ & $85.71 \%(6 / 7)$ & 0.539 \\
\hline $\begin{array}{l}\text { Right paratracheal } \\
\text { LNs }(n=116)\end{array}$ & $32.43 \%(24 / 74)$ & $31.03 \%(9 / 29)$ & $76.92 \%(10 / 13)$ & 0.850 \\
\hline The total $(n=660)$ & $12.95 \%(69 / 533)$ & $20.88 \%(19 / 91)$ & $52.78 \%(19 / 36)$ & 0.000 \\
\hline
\end{tabular}


Table 5

The size of central lymph nodes on pathology in each sublevel.

\begin{tabular}{|c|c|c|c|c|c|c|}
\hline & \multicolumn{2}{|c|}{ Long diameter of LNs } & \multirow{2}{*}{$\begin{array}{l}P \text { - } \\
\text { value }\end{array}$} & \multicolumn{2}{|c|}{ Short diameter of LNs } & \multirow{2}{*}{$\begin{array}{l}P \text { - } \\
\text { value }\end{array}$} \\
\hline & $\begin{array}{l}\text { Non-macro- } \\
\text { metastasis } \\
(\mathrm{mm})\end{array}$ & $\begin{array}{l}\text { Macro- } \\
\text { metastasis } \\
(\mathrm{mm})\end{array}$ & & $\begin{array}{l}\text { Non-macro- } \\
\text { metastasis } \\
(\mathrm{mm})\end{array}$ & $\begin{array}{l}\text { Macro- } \\
\text { metastasis } \\
(\mathrm{mm})\end{array}$ & \\
\hline $\begin{array}{l}\text { Prelaryngeal } \\
\text { LNs }(n=220)\end{array}$ & $3 \pm 0.4$ & 4 & - & $2.1 \pm 0.3$ & $2.8 \pm 0.4$ & 0.523 \\
\hline $\begin{array}{l}\text { Pretracheal LNs } \\
(n=220)\end{array}$ & $3.5 \pm 0.3$ & $5.4 \pm 0.5$ & 0.000 & $2.2 \pm 0.2$ & $4.3 \pm 0.5$ & 0.000 \\
\hline $\begin{array}{l}\text { Left } \\
\text { paratracheal } \\
\text { LNs }(n=104)\end{array}$ & $3.6 \pm 0.3$ & $4.3 \pm 0.7$ & 0.004 & $2.3 \pm 0.2$ & $3 \pm 0.4$ & 0.333 \\
\hline $\begin{array}{l}\text { Right } \\
\text { paratracheal } \\
\text { LNs }(n=116)\end{array}$ & $3.1 \pm 0.3$ & $4.9 \pm 0.4$ & 0.688 & $1.9 \pm 0.2$ & $3.5 \pm 0.3$ & 0.317 \\
\hline
\end{tabular}

\section{The ultrasound diagnostic efficacy for macro-CLNM or CLNM in each sublevel}

As revealed in Table 6 and Table 7, no matter for macro-CLNM or CLNM, the HRUS diagnostic efficacy in the prelaryngeal and pretracheal sublevels was low, with the AUC of $0.51(95 \% \mathrm{Cl} 0.44-0.58)$ and 0.55 (95\% $\mathrm{Cl} 0.48-0.62)$, respectively. Moreover, the false-negative rate of diagnosing prelaryngeal and pretracheal LNs by HRUS reached $100 \%(2 / 2)$ and $86 \%(12 / 14)$, respectively. Among them (Table 4), the proportion of LNs that were not detected by ultrasound was very high, $100 \%(2 / 2), 92 \%(11 / 12)$, respectively. Compared with the prelaryngeal-pretracheal sublevels, HRUS was more effective in diagnosing macro-CLNM in the paratracheal sublevels, with the AUC of $0.80(95 \% \mathrm{Cl} 0.71-0.87)$ in the left and $0.75(95 \% \mathrm{Cl} 0.67-0.83)$ in the right. Meanwhile, the diagnostic efficacy of macro-CLNM was also significantly higher than that of CLNM in the paratracheal sublevels ( 0.51 on the left and 0.54 on the right). When the diagnosis object was changed from CLNM to macro-CLNM, the negative predictive value (NPV) was also increased considerably. The NPV of the ultrasound diagnosis of macro-CLNM was $99 \%$ of the left paratracheal and $95 \%$ of the right side of the paratracheal. 
Table 6

Comparison of ultrasonic diagnostic efficiency for LNs macro-metastasis or metastasis in each sublevel

\begin{tabular}{|c|c|c|c|c|c|c|c|c|}
\hline & & ACC & SEN & SPE & PPV & NPV & $\begin{array}{l}\text { AUC }(95 \% \\
\text { Cl) }\end{array}$ & $P$ \\
\hline \multirow[t]{2}{*}{ Prelaryngeal LNs } & $\begin{array}{l}\text { macro- } \\
\text { metastasis }\end{array}$ & $98 \%$ & $0 \%$ & $99 \%$ & $0 \%$ & $99 \%$ & $\begin{array}{l}0.51(0.44- \\
0.58)\end{array}$ & 0.082 \\
\hline & metastasis & $92 \%$ & $0 \%$ & $99 \%$ & $0 \%$ & $94 \%$ & $\begin{array}{l}0.51(0.44- \\
0.58)\end{array}$ & 0.082 \\
\hline \multirow[t]{2}{*}{ Pretracheal LNs } & $\begin{array}{l}\text { macro- } \\
\text { metastasis }\end{array}$ & $91 \%$ & $14 \%$ & $96 \%$ & $20 \%$ & $94 \%$ & $\begin{array}{l}0.55(0.48- \\
0.62)\end{array}$ & 0.288 \\
\hline & metastasis & $80 \%$ & $5 \%$ & $96 \%$ & $20 \%$ & $82 \%$ & $\begin{array}{l}0.50(0.44- \\
0.57)\end{array}$ & 0.856 \\
\hline \multirow{2}{*}{$\begin{array}{l}\text { Left } \\
\text { paratracheal LNs }\end{array}$} & $\begin{array}{l}\text { macro- } \\
\text { metastasis }\end{array}$ & $75 \%$ & $86 \%$ & $74 \%$ & $19 \%$ & $99 \%$ & $\begin{array}{l}0.80(0.71- \\
0.87)\end{array}$ & 0.000 \\
\hline & metastasis & $59 \%$ & $31 \%$ & $71 \%$ & $32 \%$ & $70 \%$ & $\begin{array}{l}0.51(0.41- \\
0.61)\end{array}$ & 0.834 \\
\hline \multirow[t]{2}{*}{$\begin{array}{l}\text { Right paratracheal } \\
\text { LNs }\end{array}$} & $\begin{array}{l}\text { macro- } \\
\text { metastasis }\end{array}$ & $80 \%$ & $69 \%$ & $82 \%$ & $32 \%$ & $95 \%$ & $\begin{array}{l}0.75(0.67- \\
0.83)\end{array}$ & 0.000 \\
\hline & metastasis & $60 \%$ & $29 \%$ & $78 \%$ & $43 \%$ & $66 \%$ & $\begin{array}{l}0.54(0.44- \\
0.63)\end{array}$ & 0.416 \\
\hline \multicolumn{9}{|c|}{ ACC, Accuracy; SEN, Sensitivity; SPE, Specificity; } \\
\hline \multicolumn{9}{|c|}{ PPV, Positive predict value; NPV, Negative predictive value. } \\
\hline \multicolumn{9}{|c|}{ AUC, Area under the ROC curve; $\mathrm{Cl}$, Confidence interval. } \\
\hline
\end{tabular}


Table 7

Comparison between ultrasonography and pathology for central LNs sublevel-by- sublevel in PTMC

Pathology (a)

Pathology (b)

$+$

Prelaryngeal LNs $(n=220)$

$\begin{array}{lllll}+ & 0 & 3 & 0 & 3 \\ - & 2 & 215 & 14 & 203\end{array}$

Pretracheal LNs $(n=220)$

$\begin{array}{lllll}+ & 2 & 8 & 2 & 8 \\ - & 12 & 198 & 37 & 173\end{array}$

Left paratracheal LNs $(n=104)$

$\begin{array}{rrrrr}+ & 6 & 25 & 10 & 21 \\ - & 1 & 72 & 22 & 51\end{array}$

Right paratracheal LNs $(n=116)$

$\begin{array}{rrrrr}+ & 9 & 19 & 12 & 16 \\ - & 4 & 84 & 30 & 58\end{array}$

Pathology (a): Macrometastatic lymph node (the length of cancer foci $>2 \mathrm{~mm}$ ) is recorded as pathologically positive;

Pathology (b): Metastasis lymph node is recorded as pathologically positive.

\section{Discussion}

Whether patients with PTMC should routinely undergo prophylactic central neck dissection (PCND) remains controversial ${ }^{17}$. On the one hand, some scholars believe that PCND can help reduce recurrence risk and avoid secondary operations complications ${ }^{18}$. What's more, it can help stage the tumor more accurately, which is beneficial for making postoperative supplementary treatment plans (such as I-131 treatment $)^{19}$. On the other hand, there is no definite evidence to prove that PCND can improve the prognosis or prolong the survival of patients with thyroid cancer, and the expansion of the dissection scope will increase the incidence of postoperative complications ${ }^{19,20}$. Lymph node macro-metastasis is a vital risk stratification factor for PTMC patients according to the 2015 ATA guidelines ${ }^{12,21,22}$. Therefore, preoperative identification of macro-CLNM can provide a certain reference for the management of PTMC patients. 
The number and macro-metastasis rate of lymph nodes in PTMC patients are, from high to low, the right paratracheal, the left paratracheal, the pretracheal, and the prelaryngeal level. The right paratracheal level has more LNs and a higher macro-metastasis rate than the left side, which may relate to anatomy. The angle between the right RLN and the carotid artery is larger than the angle on the left side, where the RLN is parallel to the tracheoesophageal sulcus ${ }^{23}$.

This study demonstrated that the preoperative ultrasound had an extremely high false-negative rate for assessing macro-metastatic LNs in prelaryngeal-pretracheal sublevel, there were 14 macro-CLNM cases in pretracheal and prelaryngeal sublevel which were diagnosed as negative by HRUS. Among them, HRUS failed to detect any LN in 13 cases. The LNs cannot be observed by ultrasound because the prelaryngealpretracheal LNs are located between the anterior cervical muscles and the trachea. The relatively small space in this area makes the number and the size of lymph nodes small. There were only 1-2 prelaryngeal-pretracheal LNs per patient (Table 2); the average short-xis diameter of the prelaryngeal and pretracheal LNs was less than $3 \mathrm{~mm}$ and $2-4 \mathrm{~mm}$, respectively (Table 5), and even the smallest nodule detected by HRUS is only $2-3 \mathrm{~mm}^{24}$. The extremely low detective rate of HRUS in prelaryngeal-pretracheal LNs caused many false-negative cases, with the AUC of only $0.51-0.55$ (Table 6). We suggested that preoperative HRUS did not help identify the macro-CLNM in prelaryngeal-pretracheal sublevel.

Compared with the prelaryngeal-pretracheal sublevel, dissection of LNs in the paratracheal levels is more likely to cause complications such as hoarseness and hypocalcemia common in post thyroid cancer operation because of the injury of recurrent laryngeal nerve and parathyroid ${ }^{19}$. Therefore, the preoperative diagnosis of the paratracheal LNs is of great significance. The diagnostic efficacy of HRUS for CLNM in paratracheal sublevels is low, with the AUC of only $0.51-0.54$, which is consistent with the conclusions of other previous studies ${ }^{5,14}$. For macro-CLNM, the AUC of the paratracheal sublevels has been significantly improved, with 0.80 on the left and 0.75 on the right. This is probably because the diameter of macroCLNM is larger than that of the non-macro-CLNM (including no- and micro-CLNM) (Table 5), which is more easily to be detected by HRUS (Table 4). In addition, micro-CLNM has only pathological changes below $2 \mathrm{~mm}$, which is difficult to distinguish by ultrasound, while cancer foci in macro-CLNM are larger than $2 \mathrm{~mm}$, which are more likely to show abnormalities in ultrasound images. Moreover, the NPV of ultrasound diagnosis of macrometastasis in paratracheal sublevels reached $99 \%$ (left) and $95 \%$ (right). In other words, when the paratracheal sublevel is negative under HRUS, the probability of macrometastasis is extremely low, only $1 \%-5 \%$. Therefore, this study believes that preoperative HRUS has an exclusive diagnostic effect on macrometastasis in paratracheal sublevels.

It is worth mentioning that macrometastasis in prelaryngeal-pretracheal sublevel is a high risk factor for paratracheal macrometastases, with an OR value of 9.1 (95\% $\mathrm{Cl} 2.8-29.2, \mathrm{P}=0.0002)$, And among the patients with macrometastases in prelaryngeal-pretracheal sublevel, nearly $40 \%$ of them accompanied by paratracheal macrometastases (6/15). As a result, macro-CLNM in prelaryngeal-pretracheal sublevel has a certain prompting effect on paratracheal LNs macrometastasis. If PTMC patients are found with prelaryngeal-pretracheal macro-CLNM during surgery, it is necessary to be alert to ipsilateral paratracheal macro-CLNM. 
This study creatively analyzed the preoperative ultrasound and postoperative pathological results of patients with PTMC sublevel-by-sublevel, figuring out the number of LNs and macro-metastatic rate in each sublevel and discussing the diagnostic efficacy of ultrasound. Compared with the traditional rough analysis of central LNs, the ultrasound diagnostic performance obtained in this paper is more accurate and closer to the real situation. However, there are some limitations to our study. Firstly, in order to reduce the interference of contralateral central $L N$ and lateral cervical $L N$ to the analysis of ultrasound diagnostic performance in each sublevel, this retrospective study design only focus on unilateral PTMC, so there is an unavoidable selection bias. Secondly, this study can only analyze LNs sublevel-by-sublevel rather than single LN one-to-one. It remains a clinically significant topic that urgently needs research on whether combining with other imaging tests or artificial intelligence can improve diagnosis efficiency.

\section{Conclusion}

1. The number and macro-metastasis rate of lymph nodes in unilateral PTMC patients are, from high to low, the right paratracheal, the left paratracheal, the pretracheal, and the prelaryngeal level.

2. Preoperative HRUS has a high diagnostic efficiency for macro-CLNM in the ipsilateral paratracheal area (AUC is 0.80 on the left and 0.75 on the right, respectively); when the ultrasound diagnosis of the ipsilateral paratracheal area is negative, the probability of macrometastasis is extremely low.

\section{Abbreviations}

PTC

papillary thyroid cancer

PTMC

papillary thyroid microcarcinoma

CLNM

metastasis central lymph node

HRUS

high-resolution ultrasound

LNs

lymph nodes

Macro-CLNM

macro-metastatic central lymph node

Micro-CLNM

micro-metastatic central lymph node

no-CLNM

no-metastatic central lymph node

non-macro-CLNM

non-macro-metastatic central lymph node 


\section{Declarations}

\section{Ethics approval and consent to participate}

This study was approved by Sun Yat-sen University Cancer Center Institutional Review Board, and the requirement for informed consent was waived because of its retrospective nature.

\section{Consent for publication}

All the patients involved in this study were informed and the agreed that their personal data would be used for publication for the purpose of scientific research.

\section{Availability of data and materials}

This article's authenticity was validated by uploading the key raw data onto the Research Data Deposit public platform (www.researchdata.org.cn), with the Research Data Deposit number RDDA2020001813.

\section{Competing interests}

Wen Tan, Lingli Xiao, Shiwen Zhang, Haoming Zheng, Sheng Li, Yu Zhang, Ankui Yang, Jianhua Zhou, and Longzhong Liu have no conflicts of commercial interest in the subject of study and the source of any financial or material support to disclose.

\section{Funding}

This study has received funding from Natural Science Foundation of Guangdong Province, and the funding number is 2021A1515010546.

\section{Authors' contributions}

Longzhong Liu contributed substantially to the conception of the work; Longzhong Liu, Jianhua Zhou, Wen Tan, and Lingli Xiao participated in the study design; Wen Tan and Lingli Xiao drafted the work; Longzhong Liu, Wen Tan, and Lingli Xiao revised it; Shiwen Zhang, Yu Zhang, and Haoming Zheng participated in the acquisition of the data of the work and offered statistically analysis; Sheng Li and Yu Zhang offered imporment comments on the methology; Ankui Yang and Jianhua Zhou provided critical comments on the work. All the authors had read and approved the final article.

\section{Acknowledgements}

None.

\section{References}

1. Davies L, Welch HG. Current thyroid cancer trends in the United States. JAMA Otolaryngol Head Neck Surg. 2014;140(4):317-22. 
2. Ahn HS, Kim HJ, Welch HG. Korea's thyroid-cancer "epidemic"-screening and overdiagnosis. N Engl J Med. 2014;371(19):1765-7.

3. Hedinger C, Williams ED, Sobin LH. The WHO histological classification of thyroid tumors: a commentary on the second edition. Cancer. 1989;63(5):908-11.

4. Al-Qurayshi Z, Nilubol N, Tufano RP, et al. Wolf in Sheep's Clothing: Papillary Thyroid Microcarcinoma in the US. J Am Coll Surg. 2020;230(4):484-91.

5. Zhao H, Li H. Meta-analysis of ultrasound for cervical lymph nodes in papillary thyroid cancer: Diagnosis of central and lateral compartment nodal metastases. Eur J Radiol. 2019;112:14-21.

6. Pereira JA, Jimeno J, Miquel J, et al. Nodal yield, morbidity, and recurrence after central neck dissection for papillary thyroid carcinoma. Surgery. 2005;138(6):1095-100. discussion 1100 - 1091.

7. Wada N, Duh QY, Sugino K, et al. Lymph node metastasis from 259 papillary thyroid microcarcinomas: frequency, pattern of occurrence and recurrence, and optimal strategy for neck dissection. Ann Surg. 2003;237(3):399-407.

8. Ricarte-Filho J, Ganly I, Rivera M, et al. Papillary thyroid carcinomas with cervical lymph node metastases can be stratified into clinically relevant prognostic categories using oncogenic BRAF, the number of nodal metastases, and extra-nodal extension. Thyroid. 2012;22(6):575-84.

9. Teixeira G, Teixeira T, Gubert F, et al. The incidence of central neck micrometastatic disease in patients with papillary thyroid cancer staged preoperatively and intraoperatively as N0. Surgery. 2011;150(6):1161-7.

10. American Thyroid Association Guidelines Taskforce on Thyroid N. Differentiated Thyroid C, Cooper DS, et al. Revised American Thyroid Association management guidelines for patients with thyroid nodules and differentiated thyroid cancer. Thyroid 2009; 19 (11): 1167-214.

11. Leboulleux S, Rubino C, Baudin E, et al. Prognostic factors for persistent or recurrent disease of papillary thyroid carcinoma with neck lymph node metastases and/or tumor extension beyond the thyroid capsule at initial diagnosis. J Clin Endocrinol Metab. 2005;90(10):5723-9.

12. Haugen BR, Alexander EK, Bible KC, et al. 2015 American Thyroid Association Management Guidelines for Adult Patients with Thyroid Nodules and Differentiated Thyroid Cancer: The American Thyroid Association Guidelines Task Force on Thyroid Nodules and Differentiated Thyroid Cancer. Thyroid. 2016;26(1):1-133.

13. Giuliano AE, Edge SB, Hortobagyi GN. Eighth Edition of the AJCC Cancer Staging Manual: Breast Cancer. Ann Surg Oncol. 2018;25(7):1783-5.

14. Kim KE, Kim EK, Yoon JH, et al. Preoperative prediction of central lymph node metastasis in thyroid papillary microcarcinoma using clinicopathologic and sonographic features. World journal of surgery. 2013;37(2):385-91.

15. Perrier ND, Brierley JD, Tuttle RM. Differentiated and anaplastic thyroid carcinoma: Major changes in the American Joint Committee on Cancer eighth edition cancer staging manual. CA Cancer J Clin. 2018;68(1):55-63. 
16. American Thyroid Association Surgery. Working G, American Association of Endocrine S, American Academy of $\mathrm{O}-\mathrm{H}$ et al. Consensus statement on the terminology and classification of central neck dissection for thyroid cancer. Thyroid 2009; 19 (11): 1153-8.

17. Glover AR, Gundara JS, Norlen 0 , et al. The pros and cons of prophylactic central neck dissection in papillary thyroid carcinoma. Gland Surg. 2013;2(4):196-205.

18. Su H, Li Y. Prophylactic central neck dissection and local recurrence in papillary thyroid microcarcinoma: a meta-analysis. Braz J Otorhinolaryngol. 2019;85(2):237-43.

19. Son YI, Jeong HS, Baek CH, et al. Extent of prophylactic lymph node dissection in the central neck area of the patients with papillary thyroid carcinoma: comparison of limited versus comprehensive lymph node dissection in a 2-year safety study. Ann Surg Oncol. 2008;15(7):2020-6.

20. Benouaich V, Porterie J, Bouali $\mathrm{O}$, et al. Anatomical basis of the risk of injury to the right laryngeal recurrent nerve during thoracic surgery. Surg Radiol Anat. 2012;34(6):509-12.

21. Chen $Q$, Wei $T$, Wang $X L$, et al. The total number of prelaryngeal and pretracheal lymph node metastases: is it a reliable predictor of contralateral central lymph node metastasis in papillary thyroid carcinoma? J Surg Res. 2017;214:162-7.

22. Isaacs JD, Lundgren $\mathrm{Cl}$, Sidhu SB, et al. The Delphian lymph node in thyroid cancer. Ann Surg. 2008;247(3):477-82.

23. Tavares MR, Cruz JA, Waisberg DR, et al. Lymph node distribution in the central compartment of the neck: an anatomic study. Head Neck. 2014;36(10):1425-30.

24. Hughes DT, Doherty GM. Central neck dissection for papillary thyroid cancer. Cancer Control. 2011;18(2):83-8. 